\title{
Irregular Repetition Slotted ALOHA With Energy Harvesting Nodes
}

\author{
Umut Demirhan and Tolga M. Duman ${ }^{\circledR}$, Fellow, IEEE
}

\begin{abstract}
We propose an irregular repetition slotted ALOHA (IRSA) based uncoordinated random access scheme for energy harvesting (EH) nodes. Specifically, we consider the case in which each user has a battery that is recharged with harvested energy from the environment in a probabilistic manner. We analyze this scheme starting with a unit-sized battery at the nodes and extend the analysis to the case of a finite-sized battery. For both scenarios, we derive the asymptotic throughput expressions and obtain the optimized probability distributions for the number of packet replicas of the users. We demonstrate that for the case of IRSA with EH nodes, these optimized distributions perform considerably better than the alternatives, including slotted ALOHA (SA), contention resolution diversity slotted ALOHA (CRDSA), and IRSA, which do not take into account the EH process for both asymptotic and finite frame length scenarios.
\end{abstract}

Index Terms-Random access, irregular repetition slotted ALOHA, successive interference cancellation, energy harvesting, machine-to-machine (M2M) communications, asymptotic analysis.

\section{INTRODUCTION}

$\mathbf{S}$ IGNAL processing techniques enabling successive interference cancellation (SIC) in random access (RA) schemes have led the way for substantial improvement in system throughput and network stability, which become more and more important with the exponentially increasing number of Internet-of-Things (IoT) devices and the need for machineto-machine (M2M) communications. Noting that it is also of interest to devise systems that operate for a very long time, such sensing and communication nodes should be equipped with energy harvesting $(\mathrm{EH})$ capabilities to operate without the need for battery replacement. Therefore, it is essential to study ALOHA [3] and slotted ALOHA [4] based schemes providing uncoordinated RA with high throughputs using $\mathrm{EH}$ nodes.

Originating from diversity ALOHA [5], which utilizes time and frequency diversity for transmitting multiple

Manuscript received October 22, 2018; revised February 7, 2019 and May 13, 2019; accepted June 15, 2019. Date of publication July 10, 2019; date of current version September 10, 2019. This work is based on Umut Demirhan's M.S. Thesis [1] completed at Bilkent University, and it was presented in part at the IEEE International Conference on Communications (ICC), Kansas City, MO, May, 2018 [2]. The associate editor coordinating the review of this paper and approving it for publication was L. Duan. (Corresponding author: Tolga M. Duman.)

U. Demirhan is with the School of Electrical, Computer and Energy Engineering, Arizona State University, Tempe, AZ 85281 USA (e-mail: udemirhan@asu.edu).

T. M. Duman is with the Department of Electrical and Electronics Engineering, Bilkent University, 06800 Ankara, Turkey (e-mail: duman@ ee.bilkent.edu.tr).

Color versions of one or more of the figures in this paper are available online at http://ieeexplore.ieee.org.

Digital Object Identifier 10.1109/TWC.2019.2926077 copies of packets, contention resolution diversity slotted ALOHA (CRDSA) [6] considers grouping a number of slots as frames in which each user sends two copies of its packet in physical layer referred to as replicas. On the collision channel, SIC among slots is then adopted to resolve the collisions given the received signals in the entire frame. That is, a collision may be resolved by subtracting a decoded packet from the collision that includes its replica, resulting in a maximum throughput of $T \simeq 0.55$ where throughput is defined as the number of successfully resolved packets per slot [6].

After representing the SIC process explicitly on a bipartite graph, Liva proposes in [7] to vary the number of copies being transmitted according to a probability distribution resulting in irregular repetition slotted ALOHA (IRSA), and describes an iterative process to analyze the asymptotic performance of the system for a fixed repetition distribution similar to density evolution based analysis of low-density parity-check (LDPC) codes. He also shows that it is possible to optimize the repetition distribution, resulting in IRSA schemes with an asymptotic throughput close to the maximum value of 1 on the collision channel. As extensions, it is also shown that frame and slot asynchronous approaches with SIC provide significant improvements as well [8]-[10].

Stochastic nature of the energy availability at the transmitting nodes results in poor performance with standard MAC protocols. Hence, variations of the energy levels in EH communications need to be taken into account to design new medium access control schemes as addressed in many existing papers. In [11], the authors examine the stability of slotted ALOHA in an EH environment. In [12], a scheme is proposed to optimize the sum throughput of slotted ALOHA in which dynamically selected transmission power is adopted depending on the EH rate and battery. Various MAC protocols including frame ALOHA, frame slotted ALOHA (FSA) and dynamic FSA for an energy harvesting system are studied in [13]. The authors in [14] consider energy harvesting aware dynamic frame slotted ALOHA for M2M networks. Moreover, [15] examines reservation dynamic frame slotted ALOHA for M2M networks. On the other hand, there is no existing work proposing a scheme based on CRDSA or IRSA providing high throughputs with EH nodes. Given the drastically increasing number of machine type devices and their expected lifetime of operation, RA schemes with EH nodes have a tremendous potential. With this motivation, our interest in this paper is to investigate the applicability of IRSA for an EH system for the first time in the literature.

We propose a modified IRSA scheme accommodating EH nodes named as energy-harvesting irregular repetition slotted 
ALOHA (EH-IRSA). Our scheme accounts for the stochasticity of the energy level and depletion of battery, which cause poor performance for plain IRSA or CRDSA in EH environments by effectively changing the optimal repetition patterns to highly suboptimal ones. In the proposed scheme, sporadically activated users with EH capabilities are equipped with a unit or finite-sized battery. Users draw the number of replicas for their packets from a probability distribution and select specific time slots to send them across a frame, similar to IRSA. On the other hand, if a user has no energy in its selected slot, transmission cannot take place, hence the allocated slot is skipped. In our model, we assume that a unit of energy arrives with a certain probability in each slot, independently of the other slots or users. We first consider the system where the battery size is limited to one unit, and then generalize the analysis to the case of higher battery capacities. For both cases, we analyze the asymptotic throughput of the system for a fixed EH rate (which is defined as the expected number of energy arrivals in a frame), and show that finite MAC frame length throughput results conform with the theoretical (asymptotic) analysis. We utilize the derived results to find the optimal packet replica degree distributions via differential evolution for different EH rates. Our numerical results demonstrate substantial improvements with the proposed solutions in EH scenarios compared to the distributions of SA, CRDSA and the optimized distributions of IRSA not taking into account the EH process.

The paper is organized as follows. In Section II, the system model is given. The proposed EH-IRSA scheme is described and its convergence analysis is conducted in Section III. In Section IV, several optimized packet replica distributions are obtained and their performances are compared with those of CRDSA and IRSA (optimized without the EH considerations), and superiority of the proposed solutions within the EH framework is demonstrated. Conclusions are provided in Section V.

\section{SySTEM MODEL}

We consider a slotted ALOHA system in which the time slots are grouped as MAC frames (simply referred to as frames). Each frame consists of $N$ equal length slots. The total number of users that are sporadically activated (that send messages to the receiver) is denoted by $M_{t}$. The users are synchronized across the time slots and frames. Each user is activated with a probability $\pi$ for a given frame independently of activations in other frames and users. The number of active users in a frame is $M_{a}$, and the expected channel load $G$ through frames is defined as the expected number of active users per slot, i.e., $G=\mathbb{E}\left[M_{a}\right] / N=\pi M_{t} / N=M / N$.

The transmitting nodes are capable of harvesting energy from a renewable energy source and are equipped with battery of capacity $B \gamma_{i}$. For each user, in any time slot, $\gamma_{i}$ units of energy arrives with a probability $p_{c}$, which is used to recharge the battery (if it is not full) independently of the other time slots and users. ${ }^{1}$ Thus, the probability of no energy arrival in

\footnotetext{
${ }^{1}$ This energy harvesting model is common in the communications literature as an abstraction and simplification of the system (e.g., [16], [17])
}

a particular slot is $p_{n c}=1-p_{c}$. The arriving energy is lost if the battery is full. Transmission of each replica consumes $\gamma_{o}$ units of energy. For simplicity, we take $\gamma_{i}=\gamma_{o}=1$, and discuss the general case at the end of Section III.

Denoting $[k]$ as the set of integers from 1 to $k$, let $E_{s} \in$ $\{0\} \cup[B]$ be the amount of energy in the battery at the end of the $s$-th slot in a frame. If there is no transmission in slot $s+1$, we have

$$
E_{s+1}= \begin{cases}E_{s}+1 & \text { with probability } p_{c} \text { if } E_{s}<B, \\ E_{s} & \text { otherwise. }\end{cases}
$$

If there is a packet transmission at slot $(s+1)$, the amount of energy at the end of $(s+1)$-st slot is given by

$$
E_{s+1}= \begin{cases}E_{s} & \text { if } E_{s}=0 \text { or with prob. } p_{c} \text { if } E_{s}<B \\ E_{s}-1 & \text { otherwise }\end{cases}
$$

by noting that a unit of energy is consumed in the transmission.

In this paper, we only consider the energy expenditure for communications since our main interest is to introduce IRSA for the EH scenario and show the benefits that may be obtained by the proposed scheme in terms of communications. In practice, however, a certain level of energy should be kept in the battery all the time for slot synchronization ${ }^{2}$ (to enable slotted ALOHA) and other needs (e.g., sensing, keeping the receiver active, etc.). This may be considered as a separate battery from the one that is used for communication purposes.

We use the common channel model utilized in the analysis of RA schemes as in [7], namely the collision channel, and make similar assumptions throughout this work. Particularly, we assume that the receiver is able to recognize slots with a single replica, collisions and no transmission. Slots with a single replica are always successfully decoded. On the other hand, collisions are considered as non-resolvable, i.e., no information can be obtained from the collision in absence of any additional knowledge. Each packet carries the information on the slots where its copies are sent and that part of the packet is decoded independently of the whole packet that enables SIC to be employed at the receiver. ${ }^{3}$

\section{ENERGY-HARVESTING IRSA}

Before we detail the proposed scheme, let us briefly explain the original IRSA [7]. In IRSA, each user sends $k$ replicas of its packets with probability $\Lambda_{k}$ in any given frame. The probability mass function (PMF) of the number of replicas sent is represented by the polynomial $\Lambda(x)=\sum_{k=0}^{k_{\max }} \Lambda_{k} x^{k}$, which will be referred to as the repetition distribution (RD). The slots in which the replicas are sent are selected uniformly in each frame. The receiver resolves the packets through an iterative process implementing SIC by removing the other copies in different slots of successfully decoded packets. This process can be represented on a bipartite graph and it is nothing but the message passing decoding, which leads to the optimization of

\footnotetext{
${ }^{2}$ There are existing low-energy and low-complexity solutions for synchronization. For instance, in [18], the authors propose to share a low frequency reference clock that is utilized to generate the synchronized carrier signals.

${ }^{3}$ Note that to eliminate this signaling burden, random number generators of the users may be synchronized with the receiver as also indicated in [19].
} 


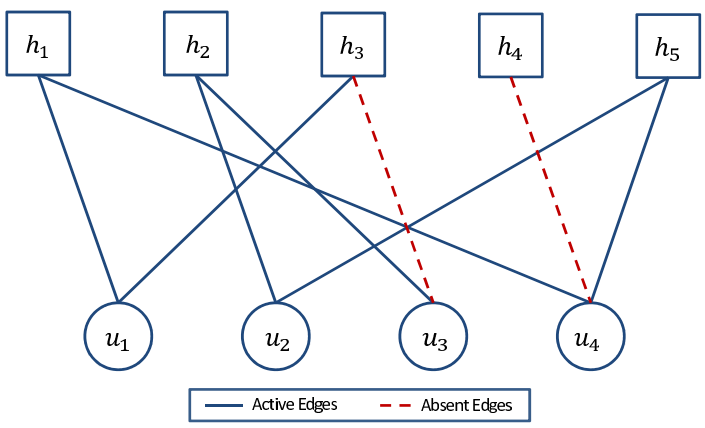

Fig. 1. An illustration of the proposed scheme with 4 users and 5 slots on a bipartite graph. Burst nodes (circles) represent users and sum nodes (squares) represent slots. Edges connect the users to their pre-determined slots.

the repetition distribution through density evolution techniques developed in the context of LDPC codes [7].

In the proposed EH-IRSA, the same protocol with IRSA is adopted along with a modification taking into account the energy state of the battery. A user transmits its replicas during its pre-determined slots only if there is energy stored in its battery, and does not transmit otherwise. In this scheme, although a user may have a certain number of replicas to send, some of these are not transmitted due to lack of energy. If a node uses an RD of $\Lambda(x)$, because of possible lack of energy, it will have a different distribution $\tilde{\Lambda}(x)$ corresponding to the probability distribution of the replicas actually being transmitted, which is referred to as the effective repetition distribution (ERD). Replicas which are not sent due to a lack of energy are called absent and the rest, that are successfully sent, are referred to as active. In Fig. 1, an example of this scheme with unit-sized battery is depicted on a bipartite graph. In this example, the number of replicas users try to send are $\{2,2,2,3\}$. However, they effectively send $\{2,2,1,2\}$ replicas. If this example were reflecting the expected number of packets and energy arrivals, the RD would be $\Lambda_{e}(x)=$ $\frac{3}{4} x^{2}+\frac{1}{4} x^{3}$ resulting in an ERD of $\tilde{\Lambda}_{e}(x)=\frac{1}{4} x+\frac{3}{4} x^{2}$.

\section{A. Convergence Analysis}

We are interested in the asymptotic throughput behavior for a load level of $G=\frac{M}{N}$ and an EH rate of $\alpha$, which is defined as the expected number of energy arrivals in a frame given by $\alpha=N p_{c}$. We let $N \rightarrow \infty$ while keeping the $\mathrm{EH}$ rate $\alpha$ and $G$ as constants, and analyze the effects of different energy arrival rates asymptotically. The corresponding density evolution analysis is similar to that of IRSA in [7], hence its details are omitted.

We define user node (UN) and sum node (SN) distributions from the edge perspective as

$$
\lambda(x) \triangleq \sum_{l} \lambda_{l} x^{l-1}, \quad \rho(x) \triangleq \sum_{l} \rho_{l} x^{l-1},
$$

respectively, where $\lambda_{l}$ and $\rho_{l}$ correspond to the probability of an edge being connected to a degree- $l$ UN and a degree- $l$ SN, respectively. Let $q_{i}$ and $p_{i}$ denote the probability of an edge not being revealed after the $i$-th iteration from a UN and an $\mathrm{SN}$, respectively. These terms can be calculated by iterating through $q_{i}=\lambda\left(p_{i-1}\right)$ and $p_{i}=1-\rho\left(1-q_{i}\right)$. Then, we obtain the resulting packet loss ratio (PLR) by converting the edge perspective probability to the node perspective probability using $P L R=\Lambda\left(p_{m}\right)$ for a large number of SIC iterations $m$, representing $m \rightarrow \infty$. Note that the PLR in this context is the ratio between the number of active users whose packets are resolved and the total number of active users since we do not ignore the users without any active replicas in the ERD to RD conversion.

The system throughput is $T=G(1-P L R)$. By utilizing a differential evolution based optimization algorithm [20], we obtain RDs that provide the maximum channel load $G^{*}$ with our choice of sufficiently low PLR values. $G^{*}$ may also be considered as the desired maximum throughput with minimal packet loss.

We emphasize that the PLR obtained using the above analysis is asymptotic, therefore the PLR values obtained by the search algorithm are also asymptotic, and the optimized distributions should be considered as approximately optimal for the finite frame length scenarios. In the following, all the PLRs in the context of analysis and optimization are asymptotic while their actual values are estimated using simulations with finite length frames.

To calculate the resulting PLR, we need to obtain $\rho(x)$ and $\lambda(x)$ polynomials, which are derived in [7] as $\lambda(x)=\frac{\Lambda^{\prime}(x)}{\Lambda^{\prime}(1)}$ and $\rho(x)=e^{-G \Lambda^{\prime}(1)(1-x)}$. Differently from [7], we utilize the ERDs instead of the RDs in the density evolution analysis of the decoding process. To clarify, we only use the active packet replicas, i.e., the absent packet replicas have no effect on the SIC process. Therefore, we further assume that the receiver is able to retrieve the knowledge of active replicas to perform the SIC operation successfully. We obtain our results based on this assumption, and clearly, if it is not achieved perfectly, there will be some performance loss. Identification of the active edges is a complicated detection problem that should be considered jointly with the code utilized in the system. For instance, the replicas can carry the slot numbers of active replicas in the previous slots and the intended transmissions in the remaining slots, which can be utilized along with the prior information about energy harvesting rates to solve this problem. Details of how this can be performed are beyond the scope of this paper.

We need to compute the ERD corresponding to a given $\mathrm{RD}$ for the analysis of the SIC process to utilize in the edge perspective probabilities $\rho(x)$ and $\lambda(x)$. Denoting the ERD by $\tilde{\Lambda}(x)=\sum_{k=0}^{k_{\max }} \tilde{\Lambda}_{k} x^{k}$, we simply change $\Lambda(x)$ to $\tilde{\Lambda}(x)$ in the equations of $\rho$ and $\lambda$ by making an approximation that active replicas are uniformly distributed across the frame. Then, we obtain $\lambda(x)=\frac{\tilde{\Lambda}^{\prime}(x)}{\tilde{\Lambda}^{\prime}(1)}$ and $\rho(x)=e^{-G \tilde{\Lambda}^{\prime}(1)(1-x)}$. In other words, we assume that the distribution of active replicas is approximately uniform to make the analysis tractable. We later show that the loss caused by this approximation is insignificant via extensive numerical examples which demonstrate the consistency of finite frame length simulation and the asymptotic analysis results.

To clarify the last point further, we note that, in IRSA, the distribution of the number of packets received on a slot is binomial because of the uniformity of the selected slots for 
TABLE I

DESCRIPTIONS OF IMPORTANT SYMBOLS

\begin{tabular}{|c|c|}
\hline Symbol & Description \\
\hline$\Lambda(x)$ & Repetition distribution (RD) \\
\hline$\tilde{\Lambda}(x)$ & Effective RD (ERD) \\
\hline$B$ & Battery capacity \\
\hline$N$ & Number of slots in a frame \\
\hline$M$ & Expected number of active users \\
\hline$\pi$ & Activity probability of a user \\
\hline$G$ & Expected channel load \\
\hline$T$ & Throughput \\
\hline$\alpha$ & EH rate (Expected energy arrivals in a frame) \\
\hline$E_{i}$ & Energy in the battery in $i$-th slot \\
\hline
\end{tabular}

sending packets. On the other hand, in EH-IRSA, this is not the case. For instance, clearly the first packet has a different probability than the others due to the sporadic activity of users as they charge their batteries while they are not active, which changes the probability of successfully sending the first packet in a frame.

Before moving on to derivations, we present a list of essential notation in Table I.

\section{B. Effective Repetition Distributions}

In this subsection, we compute the ERD for a given RD and $\mathrm{EH}$ rate. Let $\phi_{B}\left(k, l \mid E_{0}\right)$ be the asymptotic conditional probability of having $l$ active replicas out of a total of $k$ total replicas in a frame where the user has $E_{0}$ units of initial energy while the battery capacity of each user is $B$. We also define $\phi_{B}(k, l)$ as the asymptotic probability of $l$ active replicas out of $k$ replica transmission trials averaged over all possible initial energy levels. We can write

$$
\phi_{B}(k, l)=\sum_{i=0}^{B} \phi_{B}\left(k, l \mid E_{0}=i\right) \operatorname{Pr}\left(E_{0}=i\right) .
$$

We use $\Phi_{B}(x, k)$ for the polynomial representation of the PMF of the number of active replicas when a user selects $k$ replicas to be transmitted in a frame and the battery capacity is $B$. The resulting ERD and $\Phi_{B}(x, k)$ can be written as

$$
\begin{gathered}
\tilde{\Lambda}(x)=\sum_{k=0}^{k_{\max }} \Lambda_{k} \Phi_{B}(x, k), \\
\Phi_{B}(x, k)=\sum_{l=0}^{k} \phi_{B}(k, l) x^{l},
\end{gathered}
$$

respectively. From (2) and (3), we also have

$$
\tilde{\Lambda}_{l}=\sum_{k=l}^{k_{\max }} \Lambda_{k} \phi_{B}(k, l) .
$$

1) Unit-Sized Battery: We first investigate the unit-sized battery case in which a user may have energy for only one transmission or no energy. We omit battery capacity $B$ in our notation for this part since it is unity and fixed. Note that $\operatorname{Pr}\left(E_{0}=0\right)$ is the probability of not having energy at the beginning of a frame, which depends on the number of slots after the last replica in the previous frame for which the node is active. We note $\operatorname{Pr}\left(E_{0}=1\right)=1-\operatorname{Pr}\left(E_{0}=0\right)$,

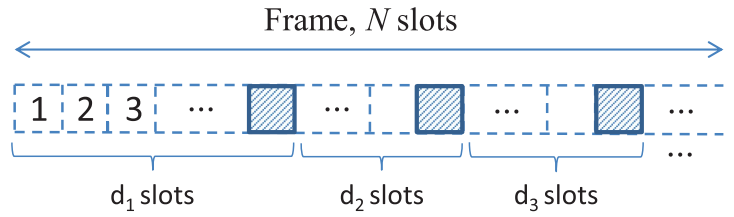

Fig. 2. A frame of a user is shown with the distance definitions where shaded squares are the selected slots to send replicas.

and in addition to the initial energy probabilities, we only need $\phi\left(k, l \mid E_{0}=0\right)$ and $\phi\left(k, l \mid E_{0}=1\right)$ to obtain $\phi(k, l)$ through (1).

Let $S_{i}$ for $i \in[k]$ be the random variable indicating that the packet $i$ is active (transmission is successful), that is,

$$
S_{i}= \begin{cases}1 & \text { if the } i \text {-th of the } k \text { replicas is active, } \\ 0 & \text { if the } i \text {-th of the } k \text { replicas is absent. }\end{cases}
$$

We also define $X_{i}=\sum_{j=1}^{i} S_{j}$ for $i \in[k]$. Note that the definition satisfies $X_{i}=X_{i-1}+S_{i}$ for $i>0$ with $X_{0}=0$.

To derive $\phi\left(k, l \mid E_{0}\right)$, we average across the possible $k$ slot selections and $l$ successful transmissions. Let $\boldsymbol{x}=$ $\left\{x_{1}, x_{2}, \ldots, x_{k}\right\}$ be the set of slot numbers that are selected to send the $k$ replicas. We also define the separations among the selected slots denoted by the vector $\boldsymbol{d}=\left\{d_{1}, d_{2}, \ldots, d_{k}\right\}$ where $d_{1}=x_{1}$ and $d_{i}=x_{i}-x_{i-1}$ for $i>1$. This is illustrated on Fig. 2 on a frame of an active user.

Clearly, $\operatorname{Pr}\left(S_{i}=0\right)=p_{n c}^{d_{i}}$ for $i \geq 1$ if $E_{0}=0$. If $E_{0}=1$, the first replica is sent, $\operatorname{Pr}\left(S_{1}=1\right)=1$ and $\operatorname{Pr}\left(S_{i}=0\right)=p_{n c}^{d_{i}}$ for $i>1$.

We use $\|\cdot\|_{1}$ to denote the $l_{1}$-norm. For every $k \in \mathbb{Z}_{+}$ and $l \in \mathbb{N}$ with $l \leq k, \mathbb{D}(k)=\left\{\boldsymbol{d} \in \mathbb{Z}_{+}^{k}:\|\boldsymbol{d}\|_{1} \leq N\right\}$ is the set of all possible slot (distance) selections, $\mathbb{B}(l)=\{\boldsymbol{b} \in$ $[k]^{l}: b_{i}<b_{j} \forall i, j \in[l]$ with $\left.i<j\right\}$ is the ordered set of all possible selection of indices of $l$ active replicas and $\operatorname{Pr}(\boldsymbol{d})$ is the probability of choosing a distance vector $\boldsymbol{d}$ (which is equivalent to choosing $k$ slots). We use the notation $\mathbb{D}$ and $\mathbb{B}$ instead of $\mathbb{D}(k)$ and $\mathbb{B}(l)$, respectively, as there should be no confusion. We also represent summation over the set of distances in the derivations and use the notation

$$
\sum_{\boldsymbol{d} \in \mathbb{D}} \triangleq \sum_{\substack{d_{1}+\cdots+d_{k} \leq N \\ d_{1}, \ldots, d_{k} \geq 1}}=\sum_{d_{k}=1}^{N-k+1} \sum_{d_{k-1}=1}^{N-k+2-d_{k}} \cdots \sum_{d_{1}=1}^{N-\sum_{i=2}^{k} d_{i}} .
$$

We can write

$$
\begin{aligned}
& \phi\left(k, l \mid E_{0}=0\right) \\
& =\lim _{N \rightarrow \infty} \sum_{\boldsymbol{d} \in \mathbb{D}} \operatorname{Pr}(\boldsymbol{d}) \operatorname{Pr}\left(X_{k}=l \mid E_{0}=0\right), \\
& =\lim _{N \rightarrow \infty} \sum_{\boldsymbol{d} \in \mathbb{D}} \operatorname{Pr}(\boldsymbol{d}) \sum_{\boldsymbol{b} \in \mathbb{B}}\left[\prod_{j \in \boldsymbol{b}} \operatorname{Pr}\left(S_{j}=1\right) \prod_{i \in[k] \backslash \boldsymbol{b}} \operatorname{Pr}\left(S_{i}=0\right)\right], \\
& =\lim _{N \rightarrow \infty} \sum_{\boldsymbol{d} \in \mathbb{D}} \operatorname{Pr}(\boldsymbol{d}) \sum_{\boldsymbol{b} \in \mathbb{B}}\left[\prod_{j \in \boldsymbol{b}}\left(1-p_{n c}^{d_{j}}\right) \prod_{i \in[k] \backslash \boldsymbol{b}} p_{n c}^{d_{i}}\right] .
\end{aligned}
$$

The summation over $\mathbb{B}$ in (5) is over all the possible $l$ active slot selections out of $k$ total selected slots for transmission. The set $\mathbb{D}$ is symmetric, hence, for any function $f^{\prime}\left(d_{1}, \ldots, d_{k}\right)$ 
and permutation $\sigma$, we have $\sum_{\boldsymbol{d} \in \mathbb{D}} f^{\prime}\left(d_{1}, \ldots, d_{k}\right)=$ $\sum_{\boldsymbol{d} \in \mathbb{D}} f^{\prime}\left(d_{\sigma(1)}, \ldots, d_{\sigma(k)}\right)$. Thanks to this property, for any selection of active and absent replicas, i.e., for any $\boldsymbol{b} \in \mathbb{B}$, the result of (5) is the same, letting us to fix $b$. There are $\left(\begin{array}{l}k \\ l\end{array}\right)$ selections of active replicas $(\boldsymbol{b} \in \mathbb{B})$, and the active users uniformly select the slots to transmit their replicas, i.e., $\operatorname{Pr}(\boldsymbol{d})=1 /\left(\begin{array}{l}N \\ k\end{array}\right)$. Thus, we can write

$$
\phi\left(k, l \mid E_{0}=0\right)=\lim _{N \rightarrow \infty} \frac{\left(\begin{array}{l}
k \\
l
\end{array}\right)}{\left(\begin{array}{l}
N \\
k
\end{array}\right)} \sum_{d \in \mathbb{D}} \prod_{j=1}^{l}\left(1-p_{n c}^{d_{j}}\right) \prod_{i=l+1}^{k} p_{n c}^{d_{i}},
$$

where we select first $l$ of $k$ replicas as active, and the rest as absent.

Note that the inner term of the summation is a polynomial in $p_{n c}$ for a given $\boldsymbol{d}$. For each term of the polynomial, the power is summation of some distances and they can be interchanged for every term individually. In other words, the result of $\sum_{\boldsymbol{d} \in \mathbb{D}} p_{n c}^{\sum_{j \in \boldsymbol{b}} d_{j}}$ is the same for all $\boldsymbol{b} \in \mathbb{B}(l)$ with $l$ being constant. Therefore, we modify (6) further by exploiting the symmetry of $\mathbb{D}$ and change $d_{i}$ and $d_{j}$ for some $i, j$ in the terms of the polynomial and write

$$
\begin{aligned}
\phi\left(k, l \mid E_{0}=0\right) \\
=\lim _{N \rightarrow \infty} \frac{\left(\begin{array}{l}
k \\
l
\end{array}\right)}{\left(\begin{array}{l}
N \\
k
\end{array}\right)} \sum_{\boldsymbol{d} \in \mathbb{D}} p_{n c}^{\sum_{i=l+1}^{k} d_{i}} \sum_{r=0}^{l}\left(\begin{array}{l}
l \\
r
\end{array}\right)(-1)^{r} p_{n c}^{\sum_{j=1}^{r} d_{j}}, \\
=\left(\begin{array}{l}
k \\
l
\end{array}\right) \sum_{r=0}^{l}\left(\begin{array}{l}
l \\
r
\end{array}\right)(-1)^{r} \lim _{N \rightarrow \infty} \frac{1}{\left(\begin{array}{l}
N \\
k
\end{array}\right)} \sum_{\boldsymbol{d} \in \mathbb{D}} p_{n c}^{\sum_{i=1}^{k-l+r} d_{i}}, \\
=\left(\begin{array}{l}
k \\
l
\end{array}\right) \sum_{r=0}^{l}\left(\begin{array}{l}
l \\
r
\end{array}\right)(-1)^{r} f(k, k-l+r),
\end{aligned}
$$

where

$$
f(k, r) \triangleq \lim _{N \rightarrow \infty} \frac{1}{\left(\begin{array}{l}
N \\
k
\end{array}\right)} \sum_{d \in \mathbb{D}} p_{n c}^{\sum_{i=1}^{r} d_{i}} .
$$

The function $f(k, r)$ can be interpreted as the asymptotic average probability of having at least $r$ absent replicas over $k$ replicas. We now seek to obtain a closed form for $f(k, r)$ without the limit and summation over different sets of distances.

Corollary 1: For given $\alpha \in \mathbb{R}_{+}$

$$
\begin{aligned}
f(k, r)= & \sum_{i=r}^{k}(-1)^{i-r} \frac{k !}{(k-i) !}\left(\begin{array}{l}
i-1 \\
i-r
\end{array}\right) \alpha^{-i} \\
& -e^{-\alpha}(-1)^{k-r} \sum_{j=k-r+1}^{k} \frac{k !}{(k-j) !}\left(\begin{array}{l}
j-1 \\
k-r
\end{array}\right) \alpha^{-j} .
\end{aligned}
$$

Proof: The result can easily be obtained from Theorem 1 that gives the result of a more general form, by setting $\boldsymbol{\delta}=\mathbf{0}$ and consequently $\|\boldsymbol{\delta}\|_{1}=0$, which is given later in the paper.

Using this corollary and (9), we can easily compute $\phi\left(k, l \mid E_{0}=0\right)$ in (1) for a given RD to calculate the ERD through (2).
For the case with unit energy at the beginning of a frame, the first replica is certainly active, i.e., $\operatorname{Pr}\left(S_{1}=1\right)=1$. We define the set $\mathbb{B}^{\prime}=\left\{\boldsymbol{b} \in \mathbb{B}: b_{1}=1\right\}$, which is a subset of set of indices in the zero initial energy case. In a complementary manner to (5) through (9), we obtain

$$
\begin{aligned}
& \phi\left(k, l \mid E_{0}=1\right) \\
& =\lim _{N \rightarrow \infty} \sum_{\boldsymbol{d} \in \mathbb{D}} \operatorname{Pr}(\boldsymbol{d}) \sum_{\boldsymbol{b} \in \mathbb{B}^{\prime}}\left[\prod_{j \in \boldsymbol{b}} \operatorname{Pr}\left(S_{j}=1\right) \prod_{i \in[k] \backslash \boldsymbol{b}} \operatorname{Pr}\left(S_{i}=0\right)\right], \\
& =\lim _{N \rightarrow \infty} \frac{\left(\begin{array}{l}
k-1 \\
l-1
\end{array}\right)}{\left(\begin{array}{l}
N \\
k
\end{array}\right)} \sum_{\boldsymbol{d} \in \mathbb{D}} \prod_{j=2}^{l}\left(1-p_{n c}^{d_{j}}\right) \prod_{i=l+1}^{k} p_{n c}^{d_{i}}, \\
& =\left(\begin{array}{c}
k-1 \\
l-1
\end{array}\right) \sum_{r=0}^{l-1}\left(\begin{array}{c}
l-1 \\
r
\end{array}\right)(-1)^{r} f(k, k-l+r) .
\end{aligned}
$$

We also need the probability of the energy states before the first slot of a frame $\operatorname{Pr}\left(E_{0}=0\right)$ and $\operatorname{Pr}\left(E_{0}=1\right) \cdot \operatorname{Pr}\left(E_{0}=0\right)$ depends on two different variables: 1) the last slot that a user tries to send a replica, and 2) the number of frames between two consecutive frames in which the user is active. We denote the expected probability of not being charged after the last trial of sending the replica in that frame as $q_{1}^{\prime}$ and the expected probability of being not charged during the frames that user is not active as $q_{2}^{\prime}$. We can write

$$
\operatorname{Pr}\left(E_{0}=0\right)=q_{1}^{\prime} q_{2}^{\prime}
$$

as these two events are independent. We form $q_{1}^{\prime}$ as

$$
q_{1}^{\prime}=\lim _{N \rightarrow \infty} \sum_{k=1}^{k_{\max }} \Lambda_{k} \frac{1}{\left(\begin{array}{l}
N \\
k
\end{array}\right)} \sum_{i=1}^{N-k+1}\left(\begin{array}{c}
N-i \\
k-1
\end{array}\right) p_{n c}^{i}
$$

which is the probability of the battery not being charged after the last trial of transmission (with the averaging over the RD), using the fact that the number of possible ways of selecting the $i$-th slot from the end of a frame as the last slot for a replica is $\left(\begin{array}{l}N-i \\ k-1\end{array}\right)$ since the remaining $k-1$ replicas are distributed across the first $N-i$ slots.

Proposition 1: The function $f(k, r)$ can also be written in the following form:

$$
f(k, r)=\lim _{N \rightarrow \infty} \frac{1}{\left(\begin{array}{l}
N \\
k
\end{array}\right)} \sum_{i=r}^{N-k+r}\left(\begin{array}{c}
i-1 \\
r-1
\end{array}\right)\left(\begin{array}{c}
N-i \\
k-r
\end{array}\right) p_{n c}^{i} .
$$

Proof: Let $i=d_{1}+\cdots+d_{r}$. The summation of $r$ distances can have the minimum value $r$ and maximum value $N-k+r$ since $k-r$ slots are needed after for the placements of remaining $k-r$ replicas. Note that the summation of the first $r$ distances and the $r$-th selected slot is $i$. Thus, the first $r-1$ replicas are needed to be sent in the first $i-1$ slots and there are $\left(\begin{array}{l}i-1 \\ r-1\end{array}\right)$ selections for the first $r$ replicas. We also need to select the slots for the remaining $k-r$ replicas, which can be sent in $N-i$ slots resulting in $\left(\begin{array}{l}N-i \\ k-r\end{array}\right)$ possibilities. The result can be obtained by summing over $i$.

We note that, by Proposition 1 , the inner summation of (14) is asymptotically equivalent to $f(k, 1)$, and we can simplify 
the expression as

$$
q_{1}^{\prime}=\sum_{k=1}^{k_{\max }} \Lambda_{k} f(k, 1)
$$

The probability of no energy arrivals during the consecutive frames for which a user is inactive can be expressed as

$$
q_{2}^{\prime}=\lim _{N \rightarrow \infty} \pi \sum_{i=0}^{\infty}\left[(1-\pi) p_{n c}^{N}\right]^{i}=\frac{\pi}{1-(1-\pi) e^{-\alpha}} .
$$

Finally, combining (13), (15) and (16), we obtain

$$
\operatorname{Pr}\left(E_{0}=0\right)=\frac{\pi}{1-(1-\pi) e^{-\alpha}} \sum_{k=1}^{k_{\max }} \Lambda_{k} f(k, 1) .
$$

2) Finite-Sized Battery: We now adopt the ERD calculation approach for a battery of non-unit capacity $(B>1)$. For the asymptotic conversion of RD to ERD in the unit-sized battery case, we average the multiplication of $l$ success and $k-l$ failure probabilities over the slot (distance) selections to find the asymptotic average probabilities of $l$ active packets over the $k$ trials as done in (5). Recall that, in the unit-sized case, at least one unit energy arrives within $d_{i}$ time slots with probability $1-p_{n c}^{d_{i}}$, and the battery is not recharged with probability $p_{n c}^{d_{i}}$. These probabilities are also success and failure probabilities of the packets. In the multi-level battery scenario, however, the state of the battery also affects the number of active packets and there can be multiple energy arrivals in a certain distance, making the success probabilities dependent on the previous ones as well as on the energy arrivals.

Let $\delta_{i}$ be the number of energy arrivals in distance $d_{i}$, and define $\delta=\left\{\delta_{1}, \delta_{2}, \ldots \delta_{k}\right\}$. For ease of exposition, we also define $e_{i}(j)$ as the probability of $i$ units of energy arriving within $d_{j}$ slots, i.e.,

$$
e_{i}(j) \triangleq \operatorname{Pr}\left(\delta_{j}=i\right)=\left(\begin{array}{c}
d_{j} \\
i
\end{array}\right) p_{c}^{i} p_{n c}^{d_{j}-i} .
$$

Similar to (5), we can write

$$
\phi\left(k, l \mid E_{0}=i\right)=\lim _{N \rightarrow \infty} \sum_{\boldsymbol{d} \in \mathbb{D}} \operatorname{Pr}(\boldsymbol{d}) \operatorname{Pr}\left(X_{k}=l \mid E_{0}=i\right) .
$$

Success of a replica depends on two random variables: 1) The amount of energy in the battery after trying to send the last replica, and 2) energy arrivals during the slots between two replicas. Moreover, to find the probability of having $l$ active replicas over $k$ replicas we need to consider the successes or failures in the previous trials. For instance, if a user has $l$ active replicas over $k$ replicas, it may have been successful in the last trial or not, depending on the number of successes in the first $k-1$ trials. If the $k$-th replica is active, either the user has energy after sending the $(k-1)$-th replica or there are energy arrivals or both. For a user to have no energy to send a replica, there must be no energy in the battery after sending the last replica and no energy arrivals during the slots between the replicas. We have

$$
\operatorname{Pr}\left(X_{i}=l \mid E_{0}=j\right)=\sum_{e=0}^{B} \operatorname{Pr}\left(X_{i}=l, E_{x_{i}}=e \mid E_{0}=j\right)
$$

where $E_{x_{i}}$ is the energy available right after the $i$-th (selected) slot. We can also write

$$
\begin{aligned}
& \operatorname{Pr}\left(X_{i}=l, E_{x_{i}}=e\right) \\
& =\sum_{l^{\prime}, e^{\prime}} \operatorname{Pr}\left(X_{i}=l, E_{x_{i}}=e \mid X_{i-1}=l^{\prime}, E_{x_{i-1}}=e^{\prime}\right) \\
& \quad \times \operatorname{Pr}\left(X_{i-1}=l^{\prime}, E_{x_{i-1}}=e^{\prime}\right)
\end{aligned}
$$

where $e \in\{0,1, \ldots, B-1\}, e^{\prime} \in\{e-1, e, \ldots, B-1\}$ with $e^{\prime} \geq 0, l \in\{0,1, \ldots, i\}$, and $l^{\prime} \in\{l-1, l\}$ with $l^{\prime} \geq 0$. We note that $\operatorname{Pr}\left(X_{i}=l, E_{x_{i}}=e \mid X_{i-1}=l^{\prime}, E_{x_{i-1}}=e^{\prime}\right)$ term depends only on the amount of energy arrivals in the slots between $(i-1)$-st and $i$-th replica trials, $\delta_{i}$. Therefore, we further simplify (18) in 3 parts:

For $e=0$;

$$
\begin{aligned}
\operatorname{Pr}\left(X_{i}=\right. & \left.l, E_{x_{i}}=e\right) \\
= & \operatorname{Pr}\left(X_{i-1}=l, E_{x_{i-1}}=0\right) \operatorname{Pr}\left(\delta_{i}=0\right) \\
& +\operatorname{Pr}\left(X_{i-1}=l-1, E_{x_{i-1}}=0\right) \operatorname{Pr}\left(\delta_{i}=1\right) \\
& +\operatorname{Pr}\left(X_{i-1}=l-1, E_{x_{i-1}}=1\right) \operatorname{Pr}\left(\delta_{i}=0\right),
\end{aligned}
$$

for $1 \leq e \leq B-2$;

$$
\begin{gathered}
\operatorname{Pr}\left(X_{i}=l, E_{x_{i}}=e\right)=\sum_{j=0}^{e+1} \operatorname{Pr}\left(X_{i-1}=l-1, E_{x_{i-1}}=j\right) \\
\times \operatorname{Pr}\left(\delta_{i}=e+1-j\right),
\end{gathered}
$$

for $e=B-1$;

$$
\begin{array}{r}
\operatorname{Pr}\left(X_{i}=l, E_{x_{i}}=e\right)=\sum_{j=0}^{B-1} \operatorname{Pr}\left(X_{i-1}=l-1, E_{x_{i-1}}=j\right) \\
\times \operatorname{Pr}\left(\delta_{i} \geq B-j\right),
\end{array}
$$

where the equations (19)-(21) are valid for $i>l \geq 1$. For $i=l \geq 1, \operatorname{Pr}\left(X_{i-1}=l, E_{x_{i-1}}=0\right)=0$ since there cannot be more successes than trials, and additionally, for $i=l=1$, $\operatorname{Pr}\left(X_{0}=0\right)=1, \operatorname{Pr}\left(X_{0}>0\right)=0$ and $\operatorname{Pr}\left(E_{x_{0}}=e\right)=$ $\operatorname{Pr}\left(E_{0}=e\right)$.

Example 1: Let $B=2$, for which there are three cases: no energy arrivals, one energy arrival and more than one energy arrivals with probabilities $e_{0}(j), e_{1}(j)$ and $e_{2}(j)$, respectively, in $d_{j}$ time slots. We consider zero initial energy $\left(E_{0}=0\right)$, and an RD for which each user sends two replicas as in CRDSA. By using (19)-(21), we calculate $\operatorname{Pr}\left(X_{2}=1, E_{x_{2}}=\right.$ $\left.0 \mid E_{0}=0\right)=p_{n c}^{d_{1}} \cdot d_{2} p_{c} p_{n c}^{d_{2}-1}+d_{1} p_{c} p_{n c}^{d_{1}-1} \cdot p_{n c}^{d_{2}}$. Similarly, $\operatorname{Pr}\left(X_{2}=1, E_{x_{2}}=1 \mid E_{0}=0\right)=p_{n c}^{d_{1}} .\left(1-d_{2} p_{c} p_{n c}^{d_{2}-1}-p_{n c}^{d_{2}}\right)$.

Therefore, for the given cases, we obtain

$$
\begin{aligned}
& \phi_{2}\left(k=2, l=1 \mid E_{0}=0\right) \\
& \quad=\lim _{N \rightarrow \infty} \operatorname{Pr}(\boldsymbol{d}) \sum_{\mathbb{D}(2)} \operatorname{Pr}\left(X_{2}=1 \mid E_{0}=0\right) \\
& \quad=\lim _{N \rightarrow \infty} \operatorname{Pr}(\boldsymbol{d}) \sum_{\mathbb{D}(2)}\left(p_{n c}^{d_{1}}-p_{n c}^{d_{1}+d_{2}}+p_{c} d_{1} p_{n c}^{d_{1}+d_{2}-1}\right) .
\end{aligned}
$$

As also observed in the example, $\operatorname{Pr}\left(X_{k}=l \mid E_{0}\right)$ is a polynomial in $p_{n c}$ similar to the unit battery case as one would expect since it is obtained by the multiplication of $e_{i}(j)$ terms. However, our original $f$ function definition does not cover 
the polynomials in the multiple battery case since the terms may also be multiplied with distances. Thus, we extend our definition of the function as follows

$$
f(k, r, \boldsymbol{\delta})=\lim _{N \rightarrow \infty} \operatorname{Pr}(\boldsymbol{d}) \sum_{\boldsymbol{d} \in \mathbb{D}(k)} \prod_{i=1}^{r}\left(\begin{array}{c}
d_{i} \\
\delta_{i}
\end{array}\right) p_{c}^{\delta_{i}} p_{n c}^{d_{i}-\delta_{i}},
$$

which indicates the asymptotic average probability of having $\delta$ as the process describing the energy arrivals between consecutive selected slots.

Theorem 1: The extended $f$ function can also be written as

$$
\begin{aligned}
& f(k, r, \boldsymbol{\delta}) \\
& =\sum_{i=r}^{k}(-1)^{i-r} \frac{k !}{(k-i) !}\left(\begin{array}{c}
\|\boldsymbol{\delta}\|_{1}+i-1 \\
i-r
\end{array}\right) \alpha^{-i} \\
& \quad-e^{-\alpha}(-1)^{k-r} \sum_{j=k-r+1-\|\boldsymbol{\delta}\|_{1}}^{k} \frac{k !}{(k-j) !}\left(\begin{array}{c}
\|\boldsymbol{\delta}\|_{1}+j-1 \\
k-r
\end{array}\right) \alpha^{-j} .
\end{aligned}
$$

Proof: The proof can be found in Appendix A.

Using the extended $f$ function and Theorem 1 , we can obtain the values of the terms of the polynomial of $p_{n c}$ without the limit or summations. For example, using the result of Theorem 1 , we readily obtain

$$
\begin{aligned}
\phi_{2}(k & \left.=2, l=1 \mid E_{0}=0\right) \\
& =f\left(2,1,\left[\begin{array}{ll}
0 & 0
\end{array}\right]\right)-f\left(2,2,\left[\begin{array}{ll}
0 & 0
\end{array}\right]\right)+f\left(2,2,\left[\begin{array}{ll}
1 & 0
\end{array}\right]\right)
\end{aligned}
$$

for Example 1 above.

In order to compute $\operatorname{Pr}\left(X_{k}=l \mid E_{0}\right)$, we can write the state transitions in a matrix form and find the final probabilities of successes and failures in a compact manner. Although there are joint success and energy states $\left(E_{x_{i}}=e, X_{i}=l\right)$, we can separate energy and success transitions by counting the failures in a polynomial form to simplify the analysis since, by definition of the energy model, we can separately consider the trials and charging, e.g., we can first update the amount of energy in the battery for the transmission by charging in $d_{i}$ slots for the $i$-th replica and then do the transmission update in the energy. To do this, we first define the vector of initial energy probabilities $\boldsymbol{W}=\left[\operatorname{Pr}\left(E_{0}=0\right) \operatorname{Pr}\left(E_{0}=1\right) \ldots \operatorname{Pr}\left(E_{0}=B\right)\right]$. We also define energy state transition matrix $\boldsymbol{T}$ in a slot separation $i$, i.e., the transition between energy levels in the battery among $d_{i}$ slots, as

$\mathbf{T}(i)=\left[\begin{array}{ccccc}e_{0}(i) & e_{1}(i) & \ldots & e_{B-1}(i) & 1-\sum_{j=0}^{B-1} e_{j}(i) \\ 0 & e_{0}(i) & \ldots & e_{B-2}(i) & 1-\sum_{j=0}^{B-2} e_{j}(i) \\ \vdots & \vdots & \ldots & \vdots & \vdots \\ 0 & 0 & \ldots & e_{0}(i) & 1-e_{0}(i) \\ 0 & 0 & \ldots & 0 & 1\end{array}\right]$

where $T_{a b}(i)=\operatorname{Pr}\left(\delta_{i}=b-a\right)$ for $b<B+1$ and $T_{a(B+1)}(i)=\operatorname{Pr}\left(\delta_{i} \geq B+1-a\right)$ with $T_{a b}(i)$ being the element of the matrix $\boldsymbol{T}(i)$ in $a$-th row and $b$-th column. Therefore, if a user has $a$ units of energy at some slot, after $d_{i}$ slots, the probability of having $b$ units of energy is $T_{a b}(i)$. Let $\boldsymbol{F}$ be the energy transition matrix for sending a replica with $F_{a b}$ being the element in the $a$-th row and $b$-th column. If there is no energy in the battery, then the replica is absent. Otherwise, one unit of energy is spent and the replica is active. We use the variable $t$ for the failure and 1 for the successes in the transition matrix of a replica transmission and write

$$
F_{a b}= \begin{cases}t, & \text { if } a=1 \text { and } b=1, \\ 1, & \text { if } a>1 \text { and } b=a-1, \\ 0, & \text { otherwise. }\end{cases}
$$

The vector of the final energy state probabilities in the polynomial form is defined as

$$
\Gamma(t)=\left[\operatorname{Pr}\left(E_{x_{k}}=0\right) \operatorname{Pr}\left(E_{x_{k}}=1\right) \ldots \operatorname{Pr}\left(E_{x_{k}}=B\right)\right] .
$$

We can write $\Gamma(t)=\boldsymbol{W} \prod_{i=1}^{k}(\boldsymbol{T}(i) \boldsymbol{F})$ and $\|\Gamma(t)\|_{1}=$ $\sum_{l=0}^{k} \operatorname{Pr}\left(X_{k}=l\right) t^{l}$ from which we can obtain $\operatorname{Pr}\left(X_{k}=l\right)$ for any $l$ using symbolic tools in terms of $e_{j}$ values by dropping the distances, and then the powers of $e_{j}$ values can be used as an input to the $f$ function in Theorem 1. Note that the initial probability matrix $\boldsymbol{W}$, which depends on the RD for continuous frames and $\alpha$ is also required for the exact analysis of the system. We can utilize the derived analysis to optimize the repetition distributions and simulate them on finite size frames to show the effectiveness of the developed approach.

Recall that we have taken $\gamma_{i}=\gamma_{o}=1$, i.e., the amount of energy arrivals and the amount consumed by transmission of each replica are identical. We note, however, that this assumption is not critical and one can extend the above analysis to the case where $\gamma_{i}$ is not necessarily the same as $\gamma_{o}$. For instance, when $\frac{\gamma_{i}}{\gamma_{o}}$ or $\frac{\gamma_{o}}{\gamma_{i}}$ is an integer, by arranging the matrix $\boldsymbol{T}$ for multiple energy arrivals if $\frac{\gamma_{i}}{\gamma_{o}}>1$ and $\boldsymbol{F}$ for multiple expenditures if $\frac{\gamma_{o}}{\gamma_{i}}>1$, the above steps can be carried out. We have not pursued this path since the resulting expressions would be significantly more complicated, and we would not obtain any significant additional insight on the performance of the system.

It is also interesting to comment on the case of infinite battery. For this case, there are several scenarios: If the average number of replicas corresponding to an optimal distribution is less than the expected value of energy arrivals, after some initial period, the system will perform as if there is no energy limitation (similar to standard IRSA) as will be shown later in Fig. 7. Otherwise, since there is no expected accumulation of energy, the proposed analysis can be utilized with a high level of battery capacity, and the optimization can be performed, as done in the numerical results section.

\section{Numerical Results}

In this section, we present several numerical examples to illustrate the results of our asymptotic analysis along with finite length simulations of the EH-IRSA scheme.

\section{A. Unit-Sized Battery Case}

We first consider the case of unit battery. Specifically, for fixed energy arrival rates and user activity probabilities, we design RDs that provide the largest channel load $G^{*}$ while keeping the PLR at a desirable value via differential evolution and the proposed convergence analysis in Section III. We limit 
TABLE II

SOME OPTIMIZED DistribUtions FOR THE UNIT BATTERY CASE

\begin{tabular}{|c|c|c|}
\hline $\boldsymbol{\alpha}$ & $\boldsymbol{\pi}$ & Distribution, $\boldsymbol{\Lambda}(\boldsymbol{x})$ \\
\hline \multirow{2}{*}{5} & 0.1 & $\Lambda_{1}(x)=0.2 x^{3}+0.04 x^{5}+0.08 x^{6}+0.19 x^{7}+0.49 x^{8}$ \\
\cline { 2 - 3 } & 1 & $\Lambda_{2}(x)=0.02 x^{4}+0.02 x^{5}+0.34 x^{7}+0.62 x^{8}$ \\
\hline
\end{tabular}

the maximum repetition degree $k_{\max }$ to 8 in our design. The resulting optimized distributions for $\alpha=5$ and $\pi=0.1,1$ are presented in Table II.

We provide asymptotic and simulation results of different distributions ${ }^{4}$ along with the optimized distribution $\Lambda_{1}(x)$ in Fig. 3 (for $\pi=0.1$ ). For the finite length simulations, we normalize the total number of users $M_{t}$ to change the expected load $G$ while keeping the activity probability $\pi$ and number of slots $N$ the same. We observe that the maximum asymptotic throughput ${ }^{5}$ for the EH system provided by $\Lambda_{L}(x)$ is about 0.58 while the newly optimized distribution $\Lambda_{1}(x)$ can offer a maximum asymptotic throughput of 0.87 , which is a substantial gain. We observe similar gains for $\pi=1$ with $\Lambda_{2}(x)$ as well. We also note that the finite length simulation results conform with the asymptotic analysis, as in the classical IRSA analysis, demonstrating the insignificance of the loss caused by the approximation made about the uniformity of the active transmissions across a frame. The classical waterfall effect of the optimization results with density evolution is also observed in the PLR graphs. We also note that the optimized distributions perform worse than the others in the region $G>G^{*}$ since an optimal RD provides the best possible distribution for the number of received replicas in a slot (especially, sufficient number of slots with a single replica), higher values of $G$ increase the number of unresolvable replicas deteriorating the system performance. ${ }^{6}$

Fig. 4 depicts the system performance with different energy harvesting rates, for a target $\mathrm{PLR}=10^{-2}$ and activity rate $\pi=1$. The results demonstrate that if the energy arrival rate is sufficiently high (e.g., $\alpha \geq 6$ for this example), the maximum throughput and the channel load reach high values. Also, optimization of the maximum channel load providing a specific target PLR (e.g., $10^{-2}$ ) is feasible above a critical value of $\alpha$, which changes slightly with different target PLR values. Below the critical value where the target PLR is not achievable, the optimization simply results in the distribution $\Lambda_{\max }=x^{8}$, which is the trivial solution where the users send their replicas as soon as possible when the energy arrival is scarce. ${ }^{7}$ Moreover, by optimizing the RDs using the proposed analysis, we can obtain higher throughputs compared to the other repetition distributions. As $\alpha$ increases, the energy scarcity is alleviated, and plain IRSA optimization $\left(\Lambda_{L}\right)$ works well while the newly optimized distributions reach high throughputs for a considerably wider range of energy arrival rate $(\alpha)$ values.

\footnotetext{
${ }^{4}$ We use $\Lambda_{L}(x)$, the 8-th order optimized distribution of the IRSA in [7] and $\Lambda_{C}(x)$, the regular-2 distribution of the classical CRDSA.

${ }^{5}$ Although we optimize for maximum channel load providing $\mathrm{PLR}=10^{-2}$, the non-optimized distributions cannot provide good $G^{*}$ values, and we make the comparison with the maximum asymptotic throughputs.

${ }^{6}$ Note that such a behavior is also observed in the standard IRSA as reported in [7].

${ }^{7}$ Recall that we take the maximum degree of a repetition distribution as 8 .
}

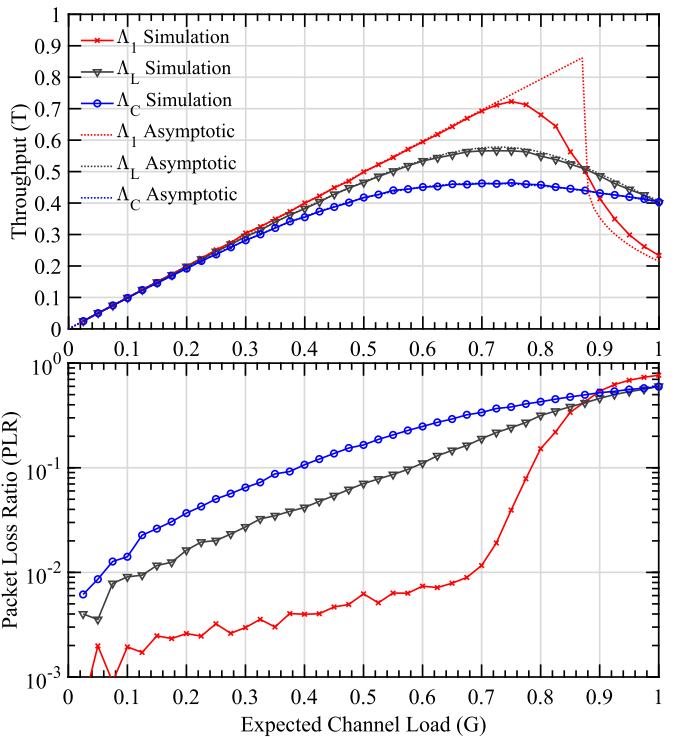

Fig. 3. The results of the simulation and analysis for energy harvesting rate $\alpha=5$, frame length $N=300$ and activity probability $\pi=0.1$.

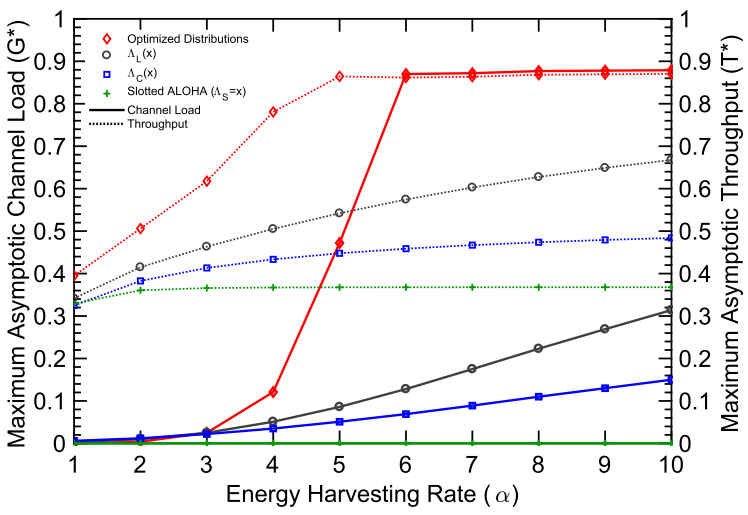

Fig. 4. Comparison of the resulting asymptotic throughputs and maximum asymptotic channel loads of the distributions optimized for different EH rates $(\alpha \in[10])$, with activity probability $\pi=1$, and $P L R=10^{-2}$.

TABLE III

SOME Optimized Distributions For THe Finite BatTery CASE

\begin{tabular}{|c|c|c|c|c|}
\hline $\boldsymbol{B}$ & $\boldsymbol{E}_{\mathbf{0}}$ & $\boldsymbol{\alpha}$ & $\boldsymbol{\pi}$ & Distribution, $\boldsymbol{\Lambda}(\boldsymbol{x})$ \\
\hline 2 & 0 & 6 & - & $\Lambda_{3}(x)=0.02 x^{2}+0.28 x^{3}+0.17 x^{4}+0.06 x^{5}+0.21 x^{6}+0.09 x^{7}+0.17 x^{8}$ \\
\hline 3 & - & 4 & 1 & $\Lambda_{4}(x)=0.14 x^{2}+0.33 x^{3}+0.10 x^{4}+0.05 x^{5}+0.19 x^{6}+0.14 x^{7}+0.05 x^{8}$ \\
\hline
\end{tabular}

\section{B. Finite-Sized Battery Case}

We now consider the scenarios with higher battery capacities, $B>1$. We optimize the RDs using the proposed analysis for different scenarios and obtain the resulting repetition distributions given in Table III for several cases.

We investigate the performance of the system in two parts. First, we obtain the optimized RD $\Lambda_{3}$ for no initial energy $\left(E_{0}=0\right)$, EH rate 6 , battery capacity 2 and a target $P L R=$ $3 \times 10^{-2}$. In Fig. 5, the corresponding asymptotic and finite frame length performances are shown along with those of other RDs. Second, we set the activity probabilities without an initial energy constraint, and resort to Monte Carlo based techniques to generate the initial energy probabilities. For a 


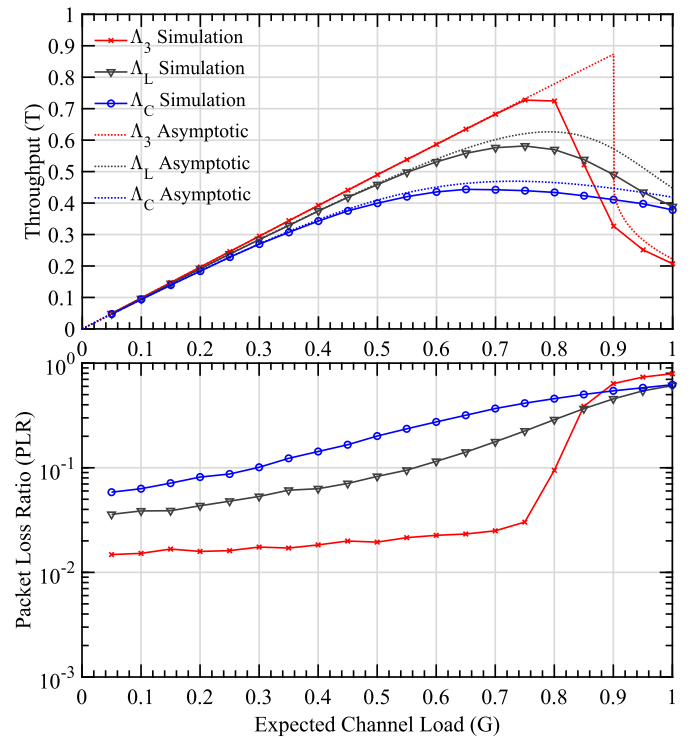

Fig. 5. Comparison of the resulting throughput of the distributions for an $\mathrm{EH}$ rate $\alpha=6$, a battery capacity $B=2$, and an initial energy $E_{0}=0$ in each frame.

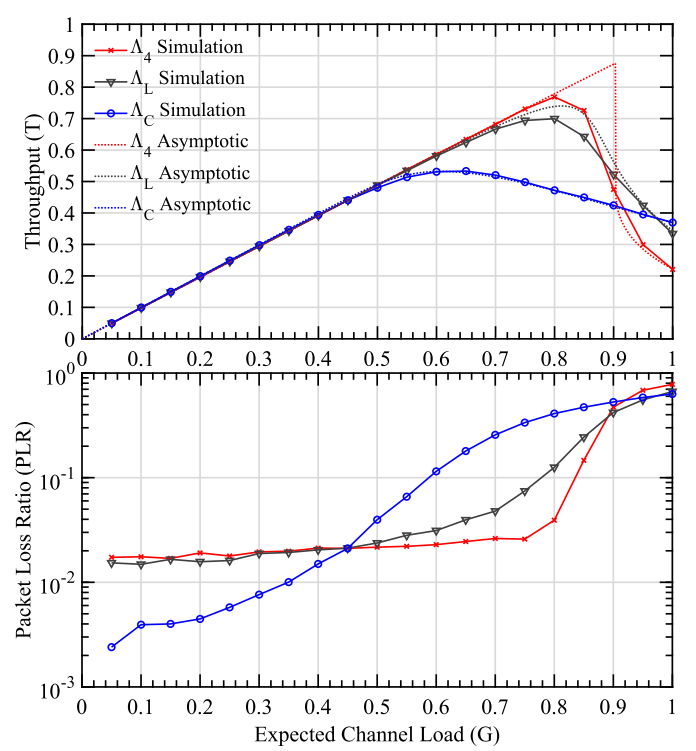

Fig. 6. Comparison of the resulting throughput of the distributions for an EH rate $\alpha=4$, an activity probability $\pi=1$, a battery capacity $B=3$.

setup with activity probability 1 , energy harvesting rate 4 , and battery capacity 3 , we optimize the RD for $P L R=3 \times 10^{-2}$ and obtain the distribution $\Lambda_{4}(x)$. We depict the corresponding asymptotic and finite frame length simulation results in Fig. 6. From both figures, we observe that substantial gains with the optimized distributions are available as in the unit battery case.

Lastly, we investigate the effects of the battery size. For a target PLR of $3 \times 10^{-2}$, we optimize the RDs for different battery capacities while $\alpha$ is fixed at 5 , and depict the resulting asymptotic channel load in Fig. 7. ${ }^{8}$ We observe that for a larger battery capacity (as more and more energy can be stored), the performance that can be gained via the proposed

\footnotetext{
${ }^{8}$ The resulting distributions are not $\Lambda_{\max }=x^{8}$ since we optimize for an $\alpha$ value above the critical level.
}

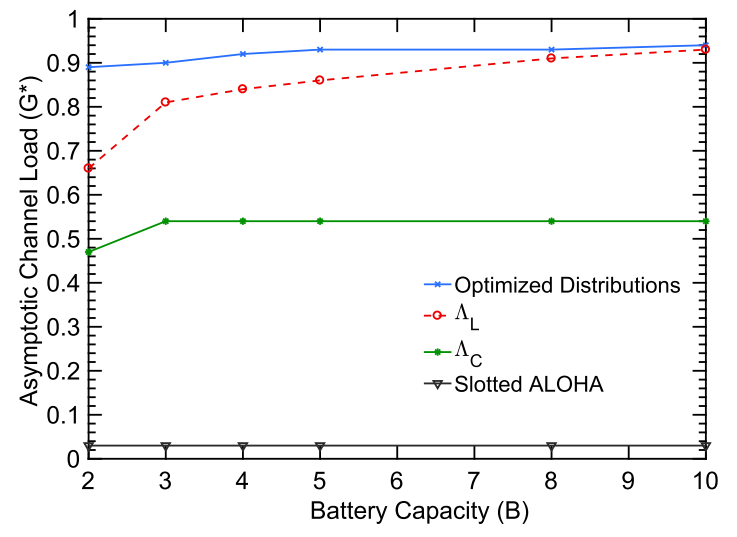

Fig. 7. Comparison of the resulting maximum asymptotic channel loads for the target $P L R=3 \times 10^{-2}$ based optimized distributions for different battery capacities given that energy harvesting rate $\alpha=5$.

optimization with $\mathrm{EH}$ decreases and the optimization without EH becomes (as in [7]) closer to optimal. Nevertheless, there is significant improvement in the limited battery scenarios.

\section{Conclusions}

With the aim of extending the high throughput performance of CRDSA, IRSA and variants of uncoordinated medium access schemes, we propose a new protocol based on IRSA for a network of EH nodes. We analyze the proposed scheme and show that IRSA-like schemes promise networks that can handle many users with EH capabilities. We optimize the repetition distributions via the developed analysis technique, and demonstrate significant improvements in throughput, especially for battery/energy limited scenarios.

The analysis technique can potentially be extended to more complex channel models, e.g., using the recent works on erasure channels in [21], [22] and fading channels in [23]. It may also be interesting to design unslotted or asynchronous [24] coded slotted ALOHA based schemes for energy harvesting systems. Another interesting direction is to study coded slotted ALOHA (CSA) [19] with EH. CSA is a general version of IRSA employing linear block codes instead of simple repetition coding, and it offers a trade-off between energy-efficiency and sustainable channel traffic. Therefore, it would be interesting to explore its energy efficiency with the use of higher rate codes for energy harvesting nodes.

\section{APPENDIX A \\ PROOF OF THEOREM 1}

To prove Theorem 1, we first present a few definitions:

Definition 1: Given functions $f(x)$ and $g(x)$ are asymptotically equivalent, i.e., $f(x) \sim g(x)$ if and only if $\lim _{x \rightarrow \infty} \frac{f(x)}{g(x)}=1$.

We denote $p_{n c}$ as $p$ in Appendix for ease of exposition and note that $p=1-\alpha / N$ for $\alpha \in \mathbb{R}$.

Remark 1: For any two asymptotically equivalent functions, i.e., $f(x) \sim g(x), p^{c} f(N)=\left(1-\frac{\alpha}{N}\right)^{c} f(N) \sim g(N)$ for all finite $\alpha, c \in \mathbb{Z}_{+}$.

Definition 2: For $x \in \mathbb{R}, n \in \mathbb{Z}_{+}$, $n$-th falling factorial of $x$ is defined as $x \underline{n}=\prod_{j=0}^{n-1}(x-j)=x ! /(x-n) !$. 
Proposition 2: Faulhaber's formula [25] for the sum of c-th power of first $n$ positive integers:

$$
\sum_{k=1}^{n} k^{c}=\frac{n^{c+1}}{c+1}+\frac{1}{2} n^{c}+\sum_{k=2}^{c} \frac{\beta_{k}}{k !} c \frac{k-1}{n} n^{c-k+1},
$$

where $\beta_{k}$ is the $k$-th Bernoulli number. Therefore, we can write

$$
\sum_{k=1}^{n} k^{c} \sim \frac{n^{c+1}}{c+1}
$$

for asymptotic equality over $n$.

Proposition 3: For some $a, b, d \in \mathbb{N}$ with $b \geq a, p \in(0,1)$ we can write

$$
\sum_{d=a}^{b} d^{\underline{x}} p^{d-x}=\xi(a, x, p)-\xi(b+1, x, p)
$$

with

$$
\xi(a, x, p) \triangleq \sum_{j=0}^{x}\left(\begin{array}{l}
x \\
j
\end{array}\right) a \frac{x-j}{a-x+j} j !(1-p)^{-(j+1)} .
$$

Proof:

$$
\begin{aligned}
\sum_{d=a}^{b} d^{x} p^{d-x} & =\sum_{d=a}^{b} \frac{d^{x}\left(p^{d}\right)}{d p^{x}}=\frac{d^{x}\left(\sum_{d=a}^{b} p^{d}\right)}{d p^{x}} \\
& =\frac{d^{x}\left(\frac{p^{a}}{1-p}\right)}{d p^{x}}-\frac{d^{x}\left(\frac{p^{b+1}}{1-p}\right)}{d p^{x}}
\end{aligned}
$$

To simplify the expression further, we need the derivative of $p^{a} \cdot(1-p)^{-1}$, let

$$
g_{1}(p)=p^{y}, \quad g_{2}(p)=(1-p)^{-1} .
$$

We denote the $i$-th derivative of $g_{1}$ and $g_{2}$ with respect to $p$ as $g_{1}^{(i)}$ and $g_{2}^{(i)}$, respectively. Then

$$
g_{1}^{(i)}(p)=y^{\underline{i}} p^{y-i}
$$

and

$$
g_{2}^{(i)}(p)=i !(1-p)^{-(i+1)} .
$$

From the general Leibnitz formula we write

$$
\left(g_{1} g_{2}\right)^{(i)}(p)=\sum_{j=0}^{i}\left(\begin{array}{l}
i \\
j
\end{array}\right) g_{1}^{(i-j)}(p) g_{2}^{j}(p)=\xi(y, i, p) .
$$

Finally, we simply combine (29) and (30) to obtain the result.

Proposition 4: From Melzak's identity [25], we have

$$
\sum_{k=0}^{n}(-1)^{k}\left(\begin{array}{l}
n \\
k
\end{array}\right) \frac{1}{y+k}=\frac{1}{\left(\begin{array}{c}
y+n \\
n
\end{array}\right)}
$$

Hence,

$$
\sum_{k=0}^{n}(-1)^{k} \frac{1}{(n-k) ! k !} \frac{1}{y+k}=\frac{(y-1) !}{(y+n) !}
$$

Proposition 5: For positive integers $m, n$, we write

$$
\sum_{i=0}^{n}\left(\begin{array}{c}
m+i \\
i
\end{array}\right)=\left(\begin{array}{c}
m+n+1 \\
n
\end{array}\right)
$$

by using the properties of binomial coefficients. We first change the first term in the summation with $\left(\begin{array}{c}m \\ 0\end{array}\right)=\left(\begin{array}{c}m+1 \\ 0\end{array}\right)$ and iteratively update the first two terms with $\left(\begin{array}{c}m \\ k\end{array}\right)+\left(\begin{array}{c}m \\ k+1\end{array}\right)=$ $\left(\begin{array}{c}m+1 \\ k+1\end{array}\right)$.

We now define an important function for the derivation of $f(k, r, \delta)$ defined in (23).

For $p \in(0,1)$ and $k, r \in \mathbb{Z}_{+}$, let $H_{k, r}:[k, \infty) \times \mathbb{N}^{k} \rightarrow[0,1]$ be a sequence of functions defined as

$$
H_{k, r}(N, \boldsymbol{\delta})=\sum_{\boldsymbol{d} \in \mathbb{D}(k)} \prod_{i=1}^{r}\left(\begin{array}{l}
d_{i} \\
\delta_{i}
\end{array}\right)(1-p)^{\delta_{i}} p^{d_{i}-\delta_{i}} .
$$

Lemma 1: $H_{k}(N, \boldsymbol{\delta}) \triangleq H_{k, k}(N, \boldsymbol{\delta})$ is asymptotically equivalent to

$$
H_{k}(N, \boldsymbol{\delta}) \sim(1-p)^{-k}-p^{N} \sum_{j=1-\|\boldsymbol{\delta}\|_{1}}^{k} \frac{(1-p)^{-j}}{(k-j) !} N^{k-j} .
$$

Proof: We can change the form of the function and obtain $H_{k}(N, \boldsymbol{\delta})$

$$
\begin{aligned}
& =\frac{1}{\delta_{1} ! \ldots \delta_{k} !} \sum_{d_{k}=\delta_{k}^{+}}^{N-\theta_{k}^{k}} d \frac{\delta_{k}}{k}(1-p)^{\delta_{k}} p^{d_{k}} \\
& \times \sum_{d_{k-1}=\delta_{k-1}^{+}}^{N-\theta_{k-1}^{k}} d \frac{\delta_{k-1}}{k-1}(1-p)^{\delta_{k-1}} p^{d_{k-1}} \ldots \sum_{d_{1}=\delta_{1}^{+}}^{N-\theta_{1}^{k}} d \frac{\delta_{1}}{1}(1-p)^{\delta_{1}} p^{d_{1}} \\
& =\frac{1}{\delta_{k} !} \sum_{d_{k}=\delta_{k}^{+}}^{N-\theta_{k}^{k}} d \frac{\delta_{k}}{k}(1-p)^{\delta_{k}} p^{d_{k}} H_{k-1}\left(N-d_{k}, \boldsymbol{\delta}\right),
\end{aligned}
$$

with

$$
\delta_{k}^{+} \triangleq \min \left(\delta_{k}, 1\right), \quad \theta_{i}^{k} \triangleq \sum_{j=i+1}^{k} d_{j}+\sum_{j=1}^{i-1} \delta_{j}^{+}
$$

for $i \in[k]$ and we take all the summations that has a starting point larger than the ending point zero, e.g., $\theta_{k}^{k}=$ $\sum_{j=1}^{k-1} \min \left(1, \delta_{j}\right)$ for $k>1$. The terms $\delta_{k}^{+}$and $\theta_{i}^{k}$ are defined for ease of exposition. Note that the second input of $H_{k, r}$ needs to have $r$ elements. We adopt $H_{k, r}(., \boldsymbol{\delta})$ for any $r \leq k$ while $\delta \in \mathbb{N}^{k}$ with an abuse of the notation, and take the first $r$ elements of $\delta$ as the input. We now prove the above statement by induction over $k$.

Base case: For $H_{1}$, we write

$H_{1}(N, \boldsymbol{\delta})$

$$
\begin{aligned}
& =\frac{1}{\delta_{1} !}(1-p)^{\delta_{1}} \sum_{d_{1}=\delta_{1}^{+}}^{N} d \frac{\delta_{1}}{1} p^{d_{1}-\delta_{1}} \\
& =\frac{1}{\delta_{1} !}(1-p)^{\delta_{1}}\left[\xi\left(\delta_{1}^{+}, \delta_{1}, p\right)-\xi\left(N+1, \delta_{1}, p\right)\right] \\
& \sim(1-p)^{-1}-\sum_{j=0}^{\delta_{1}} \frac{(1-p)^{-\left(j+1-\delta_{1}\right)}}{\left(\delta_{1}-j\right) !} N^{\delta_{1}-j} p^{N} \\
& =(1-p)^{-1}-p^{N} \sum_{j=1-\delta_{1}}^{1} \frac{(1-p)^{-j}}{(1-j) !} N^{1-j}
\end{aligned}
$$


where (39) follows from Proposition 3, (40) from Definition 1 since the summation in $\xi\left(\delta_{1}^{+}, \delta_{1}, p\right)$ is 0 for $i \neq \delta_{k}$ asymptotically, and (41) by changing the summation limits.

Inductive step: Suppose that the statement holds for $H_{k-1}$. Then for $H_{k}$, we write

$$
\begin{aligned}
H_{k}(N, \boldsymbol{\delta}) \sim & \frac{1}{\delta_{k} !} \sum_{d_{k}=\delta_{k}^{+}}^{N-\theta_{k}^{k}} d_{k}^{\delta_{k}}(1-p)^{\delta_{k}} p^{d_{k}} H_{k-1}\left(N-d_{k}, \boldsymbol{\delta}\right) \\
\sim & \frac{(1-p)^{\delta_{k}}}{\delta_{k} !} \sum_{d_{k}=\delta_{k}^{+}}^{N-\theta_{k}^{k}} d \frac{\delta_{k}}{k} p^{d_{k}}\left((1-p)^{-k+1}\right. \\
& \left.-p^{N-d_{k}} \sum_{j=1-\|\boldsymbol{\delta}\|_{1}+\delta_{k}}^{k-1} \frac{(1-p)^{-j}\left(N-d_{k}\right)^{k-1-j}}{(k-1-j) !}\right) \\
= & A_{1}+A_{2}
\end{aligned}
$$

where $\quad A_{1} \triangleq \quad \frac{(1-p)^{\delta_{k}}}{\delta_{k} !} \sum_{d_{k}=\delta_{k}^{+}}^{N-\theta_{k}^{k}} d \frac{\delta_{k}}{k} p^{d_{k}}(1-p)^{-k+1}$ and $\quad A_{2} \quad \triangleq \quad-\frac{(1-p)^{\delta_{k}}}{\delta_{k} !} \sum_{d_{k}=\delta_{k}^{+}}^{N-\theta_{k}^{k}} d \frac{\delta_{k}}{k} p^{d_{k}} p^{N-d_{k}}$

$$
\times \sum_{j=1-\|\boldsymbol{\delta}\|_{1}+\delta_{k}}^{k-1} \frac{(1-p)^{-j}\left(N-d_{k}\right)^{k-1-j}}{(k-1-j) !} .
$$

We can also write

$$
\begin{aligned}
A_{1} & =\frac{(1-p)^{\delta_{k}-k+1}}{\delta_{k} !} \sum_{d_{k}=\delta_{k}^{+}}^{N-\theta_{k}^{k}} d \frac{\delta_{k}}{k} p^{d_{k}} \\
& =A_{11}+A_{12}
\end{aligned}
$$

where we obtain two terms $A_{11}$ and $A_{12}$ after expanding (45) by Proposition 3 as

$$
A_{11} \triangleq \frac{(1-p)^{\delta_{k}-k+1}}{\delta_{k} !} \xi\left(\delta_{k}^{+}, \delta_{k}, p\right) \sim(1-p)^{-k},
$$

since the summation is not zero only for $j=\delta_{k}$ in $\xi\left(\delta_{k}^{+}, \delta_{k}, p\right)$ asymptotically, and

$$
\begin{aligned}
A_{12} & \triangleq-\frac{(1-p)^{\delta_{k}-k+1}}{\delta_{k} !} \xi\left(N-\theta_{k}^{k}+1, \delta_{k}, p\right) \\
& \sim-p^{N} \sum_{j=k-\delta_{k}}^{k} \frac{(1-p)^{-j}}{(k-j) !} N^{k-j},
\end{aligned}
$$

by changing the summation limits of $\xi\left(N-\theta_{k}^{k}+1, \delta_{k}, p\right)$ and simplification by asymptotic equality. Moreover, we have

$$
\begin{aligned}
A_{2}=-\frac{p^{N}}{\delta_{k} !} \sum_{j=1-\|\boldsymbol{\delta}\|_{1}+\delta_{k}}^{k-1} \frac{(1-p)^{\delta_{k}-j}}{(k-1-j) !} \sum_{d_{k}=\delta_{k}^{+}}^{N-\theta_{k}^{k}} d \frac{\delta_{k}}{k} \\
\times\left(N-d_{k}\right)^{k-1-j} \\
=-\frac{p^{N}}{\delta_{k} !} \sum_{j=1-\|\boldsymbol{\delta}\|_{1}+\delta_{k}}^{k-1} \frac{(1-p)^{\delta_{k}-j}}{(k-1-j) !} \sum_{d_{k}=\delta_{k}^{+}}^{N-\theta_{k}^{k}} d \frac{\delta_{k}}{k} \\
\times \sum_{i=0}^{k-1-j}\left(\begin{array}{c}
k-1-j \\
i
\end{array}\right)(-1)^{i} d_{k}^{i} N^{k-1-j-i}
\end{aligned}
$$

$$
\begin{aligned}
& =-\frac{p^{N}}{\delta_{k} !} \sum_{j=1-\|\boldsymbol{\delta}\|_{1}+\delta_{k}}^{k-1}(1-p)^{\delta_{k}-j} \sum_{i=0}^{k-1-j} \frac{(-1)^{i}}{(k-1-j-i) ! i !} \\
& \times N^{k-1-j-i} \sum_{d_{k}=\delta_{k}^{+}}^{N-\theta_{k}^{k}} d \frac{\delta_{k}}{k} d_{k}^{i} \\
& \sim-\frac{p^{N}}{\delta_{k} !} \sum_{j=1-\|\boldsymbol{\delta}\|_{1}+\delta_{k}}^{k-1}(1-p)^{\delta_{k}-j} N^{k+\delta_{k}-j} \\
& \times \sum_{i=0}^{k-1-j} \frac{(-1)^{i}}{(k-1-j-i) ! i !\left(\delta_{k}+i+1\right)} \\
& \sim-p^{N} \sum_{j=1-\|\delta\|_{1}}^{k-1-\delta_{k}} \frac{1}{(k-j) !}(1-p)^{-j} N^{k-j}
\end{aligned}
$$

where (49)-(53) follow from a rearrangement of the terms, binomial expansion of $\left(N-d_{k}\right)^{k-1-j}$, another rearrangement of the terms, Proposition 2 on the summation over $d_{k}^{\delta_{k}+i}$, and Proposition 4 with a change of the summations limits, respectively. The summation $A_{11}+A_{12}+A_{2}$ in (47), (48) and (53) gives the final result proving the statement.

Lemma 2: $H_{k, r}(N, \boldsymbol{\delta})$ is asymptotically equivalent to

$$
\begin{aligned}
& H_{k, r}(N, \boldsymbol{\delta}) \\
& \sim \sum_{i=r}^{k}(-1)^{i-r} \frac{\left(\begin{array}{c}
\|\boldsymbol{\delta}\|_{1}+i-1 \\
i-r
\end{array}\right)}{(k-i) !}(1-p)^{-i} N^{k-i}
\end{aligned}
$$

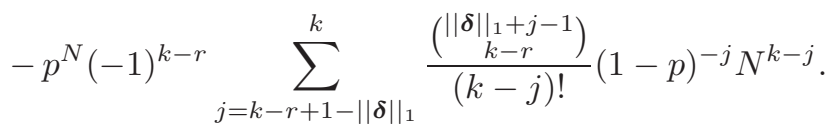

Proof: By the definition of the function (Definition 3), we can write

$$
\begin{aligned}
H_{k, r}(N, \boldsymbol{\delta}) & =\sum_{d_{k}=\delta_{k}^{+}}^{N-\theta_{k}^{k}} \cdots \sum_{d_{r+1}=\delta_{r+1}^{+}}^{N-\theta_{r+1}^{k}} H_{r, r}\left(N-\sum_{i=r+1}^{k} d_{i}, \boldsymbol{\delta}\right) \\
& =\sum_{d_{k}=\delta_{k}^{+}}^{N-\theta_{k}^{k}} H_{k-1, r}\left(N-d_{k}, \boldsymbol{\delta}\right) .
\end{aligned}
$$

Again, we can prove the statement by induction.

Base case: Note that by definition $H_{r, r}(x)=H_{r}(x)$. If we replace every instance of $k$ with $r$ in (54), we get the statement (34) of Lemma 2, which was already proved.

Inductive step: Suppose that the statement holds for $H_{k-1, r}$. Now we write

$$
H_{k, r}(N, \boldsymbol{\delta})=\sum_{d_{k}=\delta_{k}^{+}}^{N-\theta_{k}^{k}} H_{k-1, r}\left(N-d_{k}, \boldsymbol{\delta}\right)=C_{1}+C_{2},
$$

by expanding $H_{k-1, r}\left(N-d_{k}, \boldsymbol{\delta}\right)$ with the statement of the lemma, where

$C_{1}$

$$
\triangleq \sum_{d_{k}=\delta_{k}^{+}}^{N-\theta_{k}^{k}} \sum_{i=r}^{k-1}(-1)^{i-r} \frac{\left(\begin{array}{l}
\|\boldsymbol{\delta}\|_{1}+i-1 \\
i-r
\end{array}\right)}{(k-1-i) !}(1-p)^{-i}\left(N-d_{k}\right)^{k-1-i}
$$




$$
\begin{aligned}
& =\sum_{i=r}^{k-1}(-1)^{i-r} \frac{\left(\begin{array}{c}
\|\boldsymbol{\delta}\|_{1}+i-1 \\
i-r
\end{array}\right)}{(k-1-i) !}(1-p)^{-i} \sum_{d_{k}=\delta_{k}^{+}}^{N-\theta_{k}^{k}}\left(N-d_{k}\right)^{k-1-i} \\
& \sim \sum_{i=r}^{k-1}(-1)^{i-r} \frac{\left(\begin{array}{c}
\|\boldsymbol{\delta}\|_{1}+i-1 \\
i-r
\end{array}\right)}{(k-i) !}(1-p)^{-i} N^{k-i}
\end{aligned}
$$

by changing the summation over $d_{k}$ to $d_{k}^{\prime}=N-d_{k}$ in (58) and using Proposition 2 for (59), and

$$
\begin{aligned}
C_{2} \triangleq & (-1)^{k-r} \sum_{j=k-r-\|\boldsymbol{\delta}\|_{1}}^{k-1} \frac{\left(\begin{array}{c}
\|\boldsymbol{\delta}\|_{1}+j-1 \\
k-1-r
\end{array}\right)}{(k-1-j) !}(1-p)^{-j} \\
& \times \sum_{d_{k}=\delta_{k}^{+}}^{N-\theta_{k}^{k}}\left(N-d_{k}\right)^{k-1-j} p^{N-d_{k}} \\
\sim & (-1)^{k-r} \sum_{j=k-r-\|\boldsymbol{\delta}\|_{1}}^{k-1} \frac{\left(\begin{array}{c}
\|\boldsymbol{\delta}\|_{1}+j-1 \\
k-1-r
\end{array}\right)}{(k-1-j) !}(1-p)^{-j} \\
& \times\left[\xi\left(\theta_{k}^{k}, k-1-j, p\right)-\xi\left(N-\delta_{k}^{+}+1, k-1-j, p\right)\right] \\
= & C_{21}+C_{22}
\end{aligned}
$$

where we obtain (61) by first changing the summation over $d_{k}$ to $d_{k}^{\prime}=N-d_{k}$ and using Proposition 3 . We note that $C_{21}$ and $C_{22}$ are the terms obtained by multiplying the first part in (61) over the terms in the parenthesis, which are $\xi\left(\theta_{k}^{k}, k-1-j, p\right)$ and $-\xi\left(N-\delta_{k}^{+}+1, k-1-j, p\right)$, respectively. Now we have

$$
\begin{array}{r}
C_{21} \sim(-1)^{k-r} \sum_{j=k-r-\|\boldsymbol{\delta}\|_{1}}^{k-1} \sum_{a=0}^{k-1-j} \frac{\left(\begin{array}{c}
\|\boldsymbol{\delta}\|_{1}+j-1 \\
k-1-r
\end{array}\right)}{(k-1-j-a) !} \\
\times\left(\theta_{k}^{k}\right) \frac{k-1-j-a}{(1-p)^{-(a+j+1)}} \\
=(-1)^{k-r} \sum_{a=k-r-\|\boldsymbol{\delta}\|_{1}}^{k-1} \sum_{j=k-r-\|\boldsymbol{\delta}\|_{1}}^{a} \frac{\left(\begin{array}{c}
\|\boldsymbol{\delta}\|_{1}+j-1 \\
k-1-r
\end{array}\right)}{(k-1-a) !} \\
\times\left(\theta_{k}^{k}\right) \frac{k-1-a}{k-1-p)^{-(a+1)}}(1-1)^{k-r}(1-p)^{-k} \sum_{j=k-r-\|\boldsymbol{\delta}\|_{1}}^{k-1}\left(\begin{array}{c}
\|\boldsymbol{\delta}\|_{1}+j-1 \\
k-1-r
\end{array}\right) \\
=(-1)^{k-r}(1-p)^{-k}\left(\begin{array}{c}
\|\boldsymbol{\delta}\|_{1}+k-1 \\
k-r
\end{array}\right)
\end{array}
$$

where (64)-(66) are derived by a change of variables, asymptotic simplification with only $a=k-1$ from the first summation, and Proposition 5, respectively. Moreover,

$$
\begin{array}{r}
C_{22} \sim-p^{N}(-1)^{k-r} \sum_{j=k-r-\|\boldsymbol{\delta}\|_{1}}^{k-1} \sum_{b=0}^{k-1-j} \frac{\left(\begin{array}{c}
\|\boldsymbol{\delta}\|_{1}+j-1 \\
k-1-r
\end{array}\right)}{(k-1-j-b) !} \\
\times N^{k-1-j-b}(1-p)^{-(b+j+1)}
\end{array}
$$

$$
\begin{gathered}
=-p^{N}(-1)^{k-r} \sum_{b=k-r-\|\boldsymbol{\delta}\|_{1}}^{k-1} \frac{N^{k-1-b}(1-p)^{-(b+1)}}{(k-1-b) !} \\
\times \sum_{j=k-r-\|\boldsymbol{\delta}\|_{1}}^{b}\left(\begin{array}{c}
\|\boldsymbol{\delta}\|_{1}+j-1 \\
k-1-r
\end{array}\right) \\
=-p^{N}(-1)^{k-r} \sum_{b=k-r+1-\|\boldsymbol{\delta}\|_{1}}^{k} \frac{\left(\begin{array}{l}
\|\boldsymbol{\delta}\|_{1}+b-1 \\
k-r
\end{array}\right)}{(k-b) !}(1-p)^{-b} N^{k-b},
\end{gathered}
$$

where (67)-(69) are obtained by asymptotic equality, a change of summation variables, and Proposition 5 with a change in the summation limits, respectively. $C_{1}+C_{21}+C_{22}$ in (59), (66), and (69) results in (54) completing the proof.

Finally, we can prove Theorem 1 as follows:

Proof: Note that $f(k, r, \boldsymbol{\delta})=\lim _{N \rightarrow \infty} \operatorname{Pr}(\boldsymbol{d}) H_{k, r}(N, \boldsymbol{\delta})$. Therefore, we multiply the result of Lemma 3, which is also asymptotic in $N$, with $\left(1 /\left(\begin{array}{l}N \\ k\end{array}\right)\right)$ and take the limit in a straightforward manner to complete the proof.

\section{REFERENCES}

[1] U. Demirhan, "Irregular repetition slotted ALOHA with energy harvesting nodes," M.S. Thesis, Dept. of Elect. Electron. Eng., Bilkent University, Ankara, Turkey, 2017.

[2] U. Demirhan and T. M. Duman, "Energy-harvesting irregular repetition slotted ALOHA with unit-sized battery," in Proc. IEEE Int. Conf. Commun. (ICC), Kansas City, MO, USA, May 2018, pp. 1-6.

[3] N. Abramson, "THE ALOHA SYSTEM: Another alternative for computer communications," in Proc. Joint Comput. Conf. AFIPS, Nov. 1970, pp. 281-285.

[4] L. G. Roberts, "ALOHA packet system with and without slots and capture," ACM SIGCOMM Comput. Commun. Rev., vol. 5, no. 2, pp. 28-42, 1975.

[5] G. Choudhury and S. Rappaport, "Diversity ALOHA-A random access scheme for satellite communications," IEEE Trans. Commun., vol. 31, no. 3, pp. 450-457, Mar. 1983.

[6] E. Casini, R. De Gaudenzi, and O. del Rio Herrero, "Contention resolution diversity slotted ALOHA (CRDSA): An enhanced random access scheme for satellite access packet networks," IEEE Trans. Wireless Commun., vol. 6, no. 4, pp. 1408-1419, Apr. 2007.

[7] G. Liva, "Graph-based analysis and optimization of contention resolution diversity slotted ALOHA," IEEE Trans. Commun., vol. 59, no. 2 , pp. 477-487, Feb. 2011.

[8] C. Kissling, "Performance enhancements for asynchronous random access protocols over satellite," in Proc. IEEE Int. Conf. Commun. (ICC), Kyoto, Japan, Jun. 2011, pp. 1-6.

[9] C. Stefanović and P. Popovski, "ALOHA random access that operates as a rateless code," IEEE Trans. Commun., vol. 61, no. 11, pp. 4653-4662, Nov. 2013.

[10] R. D. Gaudenzi, O. D. R. Herrero, G. Acar, and E. G. Barrabés, "Asynchronous contention resolution diversity ALOHA: Making CRDSA truly asynchronous," IEEE Trans. Wireless Commun., vol. 13, no. 11, pp. 6193-6206, Nov. 2014.

[11] J. Jeon and A. Ephremides, "On the stability of random multiple access with stochastic energy harvesting," IEEE J. Sel. Areas Commun., vol. 33, no. 3, pp. 571-584, Mar. 2015.

[12] M. Moradian and F. Ashtiani, "Sum throughput maximization in a slotted Aloha network with energy harvesting nodes," in Proc. IEEE Wireless Commun. Netw. Conf. (WCNC), Apr. 2014, pp. 1585-1590.

[13] F. Iannello, O. Simeone, and U. Spagnolini, "Medium access control protocols for wireless sensor networks with energy harvesting," IEEE Trans. Commun., vol. 60, no. 5, pp. 1381-1389, May 2012. 
[14] S. Wu, Y. Chen, K. K. Chai, F. Vazquez-Gallego, and J. Alonso-Zarate, "Analysis and performance evaluation of dynamic frame slotted-ALOHA in wireless machine-to-machine networks with energy harvesting," in Proc. IEEE Globecom Workshops (GC Wkshps), Austin, Texas, USA, Dec. 2014, pp. 1081-1086.

[15] F. Vazquez-Gallego, J. Alonso-Zarate, and L. Alonso, "Reservation dynamic frame slotted-ALOHA for wireless M2M networks with energy harvesting," in Proc. IEEE Int. Conf. Commun. (ICC), Jun. 2015, pp. 5985-5991.

[16] D. Shaviv, P.-M. Nguyen, and A. Özgür, "Capacity of the energyharvesting channel with a finite battery," IEEE Trans. Inf. Theory, vol. 62, no. 11, pp. 6436-6458, Nov. 2016.

[17] K. Tutuncuoglu, O. Ozel, A. Yener, and S. Ulukus, "The binary energy harvesting channel with a unit-sized battery," IEEE Trans. Inf. Theory, vol. 63, no. 7, pp. 4240-4256, Jul. 2017.

[18] O. Abari, H. Rahul, D. Katabi, and M. Pant, "AirShare: Distributed coherent transmission made seamless," in Proc. IEEE Conf. Comput. Commun. (INFOCOM), Apr. 2015, pp. 1742-1750.

[19] E. Paolini, G. Liva, and M. Chiani, "Coded slotted ALOHA: A graphbased method for uncoordinated multiple access," IEEE Trans. Inf. Theory, vol. 61, no. 12, pp. 6815-6832, Dec. 2015.

[20] R. Storn and K. Price, "Differential evolution-A simple and efficient heuristic for global optimization over continuous spaces," J. Global Optim., vol. 11, no. 4, pp. 341-359, Dec. 1997.

[21] M. Ivanov, F. Brännström, A. Graell i Amat, and P. Popovski, "Error floor analysis of coded slotted ALOHA over packet erasure channels," IEEE Commun. Lett., vol. 19, no. 3, pp. 419-422, Mar. 2015.

[22] Z. Sun, Y. Xie, J. Yuan, and T. Yang, "Coded slotted ALOHA for erasure channels: Design and throughput analysis," IEEE Trans. Commun., vol. 65, no. 11, pp. 4817-4830, Nov. 2017.

[23] F. Clazzer, E. Paolini, I. Mambelli, and C. Stefanović, "Irregular repetition slotted ALOHA over the Rayleigh block fading channel with capture," in Proc. IEEE Int. Conf. Commun. (ICC), May 2017, pp. 1-6.

[24] E. Sandgren, A. G. I. Amat, and F. Brännström, "On frame asynchronous coded slotted ALOHA: Asymptotic, finite length, and delay analysis," IEEE Trans. Commun., vol. 65, no. 2, pp. 691-704, Feb. 2017.

[25] J. Quaintance and H. W. Gould, Combinatorial Identities for Stirling Numbers. Cleveland, OH, USA: World Scientific, 2015.

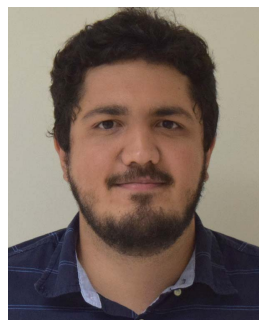

Umut Demirhan received the B.S. and M.S. degrees in electrical and electronics engineering from Bilkent University, Ankara, Turkey, in 2015 and 2017, respectively. $\mathrm{He}$ is currently pursuing the $\mathrm{Ph} . \mathrm{D}$. degree in electrical engineering at Arizona State University, Tempe, AZ, USA. His current research interests include various topics in machine learning, optimization, and wireless communications with a particular focus on machine learning applications at the wireless edge.

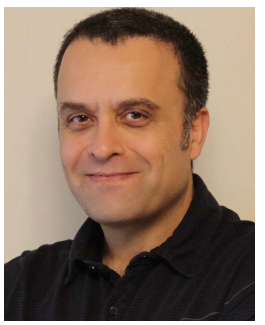

Tolga M. Duman (S'95-M'98-SM'03-F'11) received the B.S. degree from Bilkent University, Ankara, Turkey, in 1993, and the M.S. and Ph.D. degrees from Northeastern University, Boston, MA, USA, in 1995 and 1998, respectively, all in electrical engineering.

$\mathrm{He}$ is currently a Professor with the Electrical and Electronics Engineering Department, Bilkent University. Prior to joining Bilkent University in 2012, he was a Professor with the School of ECEE at Arizona State University. His curren research interests are in systems, with particular focus on communication and signal processing, including wireless and mobile communications, coding/modulation, coding for wireless communications, data storage systems, and underwater acoustic communications. He was a recipient of the National Science Foundation CAREER Award and the IEEE Third Millennium Medal. He has served as an Editor for the IEEE TRANSACTIONS ON WIRELESS COMMUNICATIONS from 2003 to 2008, the IEEE COMmunications SuRVEYS AND TUTORIALS from 2002 to 2007, the IEEE TRANSACTIONS ON COMMUNICATIONS from 2007 to 2012, and Physical Communication (Elsevier) from 2010 to 2016. He has been the Coding and Information Theory Area Editor of the IEEE TRANSACTIONS ON COMMUNICATIONS since 2011, an Editor of the IEEE TRANSACTIONS on Wireless Communications, and the Editor-in-Chief of Physical Communication (Elsevier) since 2016. 